\title{
On $\Lambda^{\lambda}$-Homeomorphisms In Topological Spaces
}

\author{
M. Gilbert Rani ${ }^{1}$ and S. Pious Missier ${ }^{2}$ \\ 1 Post Graduate Department of Mathematics, V.O.C. College, Tuticorin, India; ${ }^{2}$ Post Graduate \\ Department of Mathematics, V.O.C. College, Tuticorin, India \\ E-mail: gil.anto@yahoo.in
}

Received: February 7, 2011 / Accepted: March 23, 2011

\begin{abstract}
In this paper, we first introduce a new class of closed map called $\Lambda^{\lambda}$-closed map. Moreover, we introduce a new class of homeomorphism called $\Lambda^{\lambda}$ - Homeomorphism, which are weaker than homeomorphism. We also introduce $\Lambda^{\lambda^{*}}$ - Homeomorphisms and prove that the set of all $\Lambda^{\lambda}$ - Homeomorphisms form a group under the operation of composition of maps. 2000 Math Subject Classification: 54C08, 54D05.
\end{abstract}

Keywords and Phrases: $\Lambda^{\lambda}$-closed sets, $\Lambda^{\lambda}$-open sets, $\Lambda^{\lambda}$ - continuous function, $\Lambda^{\lambda}$ - irresolute map, $\Lambda$-g-closed set, $\Lambda^{\lambda}$-homeomorphism, $\Lambda^{\lambda^{*}}$ - homeomorphism. 


\section{Introduction}

The notion homeomorphism plays a very important role in topology. By definition, a homeomorphism between two topological spaces $X$ and $Y$ is a bijective map $f: X \rightarrow Y$ when both and $f$ and $f^{-1}$ are continuous. Maki. et al. [5] introduced

$\mathrm{g}$-homeomorphisms and gc-homeomorphisms in topological spaces.

In this paper, we first introduce $\Lambda^{\lambda}$ - closed maps in topological spaces and then we introduce and study $\Lambda^{\lambda}$-homeomorphisms, which are weaker than homeomorphisms. We also introduce $\Lambda^{\lambda^{*}}$ - homeomorphisms. It turns out that the set of all $\Lambda^{\lambda^{*}}$-homeomorphisms forms a group under the operation composition of functions.

\section{Preliminaries}

Throughout this paper $(X, \tau)$ and $(Y, \sigma)$ represent topological spaces on which no separation axioms are assumed unless otherwise mentioned. For a subset $A$ of a space $(X, \tau), C l(A), \operatorname{Int}(A)$ ant $X-A$ denote the closure of $A$, the interior of $A$ and the complement of $A$ in $X$, respectively.

We recall the following definitions and some results, which are used in the sequel.

Definition 2.1: A subset $A$ of a space $(X, \tau)$ is called:-

(1) $\lambda$ - closed [1] if $A=B \cap C$, where $B$ is a $\Lambda$ - set and $C$ is a closed set.

The complement of $\lambda$-closed set is called a $\lambda$ - open set.

(2) $\Lambda$-g-closed [2] if $\mathrm{Cl}_{\lambda}(\mathrm{A}) \subseteq U$, wherever $\mathrm{A} \subseteq U, U$ is $\lambda$-open, where $\mathrm{Cl}_{\lambda}$ (A)

[4] is called the $\lambda$ - Closure of $A$. The complement of $\Lambda$-g-closed set is called a

$\Lambda$-g-closed-open set.

The family of all $\lambda$ - open subsets of a space $(X, \tau)$ shall be denoted by $\lambda O(X, \tau)$.

\section{Definition 2.2:}

A subset $A$ of a space $(X, \tau)$ is called a generalized closed (briefly g-closed) set [6] if $\mathrm{Cl}(A) \subset U$ whenever $A \subset U$ and $U$ is open in $(X, \tau)$.

Definition 2.5: A function $f:(\mathrm{X}, \tau) \rightarrow(\mathrm{Y}, \sigma)$ is called:

(1) $\lambda$-continuous $[1]$ if $f^{-1}(\mathrm{~V})$ is $\lambda$-open in $(\mathrm{X}, \tau)$, for every open set $\mathrm{V}$ in $(\mathrm{Y}, \sigma)$. 
(2) $\lambda$-irresolute [3] if $f^{-1}(\mathrm{~V})$ is $\lambda$-open in $(\mathrm{X}, \tau)$, for every $\lambda$ open set $\mathrm{V}$ in $(\mathrm{Y}, \sigma)$.

(3) g-continuous [6] if $f^{-1}(\mathrm{~V})$ is g-closed in $(\mathrm{X}, \tau)$ for every $\mathrm{g}$-closed set $\mathrm{V}$ in $(\mathrm{Y}, \sigma)$.

(4) gc-irresolute [6] if $f^{-1}(\mathrm{~V})$ is g-closed in $(\mathrm{X}, \tau)$ for every g-closed set $\mathrm{V}$ in $(\mathrm{Y}, \sigma)$.

(5) $\lambda$-closed [3] if $f(\mathrm{~V})$ is $\lambda$-closed in $(\mathrm{Y}, \sigma)$, for every closed set $\mathrm{V}$ in $(\mathrm{X}, \tau)$.

Definition 2.6: A function $f:(\mathrm{X}, \tau) \rightarrow(\mathrm{Y}, \sigma)$ is called g-open [6] if $f(\mathrm{~V})$ is g-open in $(Y, \sigma)$, for every g-open set $V$ in $(X, \tau)$.

Definition 2.7: A bijective function $f:(\mathrm{X}, \tau) \rightarrow(\mathrm{Y}, \sigma)$ is called a:

(1) generalized homeomorphism (briefly g-homeomorphism) [7] if $f$ is both g-continuous and gopen.

(2) gc-homeomorphism [7] if both $f$ and $f^{-1}$ are gc-irresolute maps.

\section{3. $\Lambda^{\lambda}$-Closed Sets}

Definition 3.1: A subset $A$ of a topological space $(X, \tau)$ is called :

(1) $\Lambda_{*}^{\lambda}$ - set if $A=A^{\Lambda_{*}{ }_{*}}$, where $A^{\Lambda_{*}^{\lambda}}=\cap\{B: B \supset A, B \in \lambda o(X, \tau)\}$

(2) $\Lambda^{\lambda}$ - closed set if $A=L \cap F$, where $L$ is $\Lambda_{*}^{\lambda}$ - set and $F$ is $\lambda$ - closed.

The complement of $\Lambda_{*}^{\lambda}$-set and $\Lambda^{\lambda}$-closed set is $V_{*}^{\lambda}$-set and $\Lambda^{\lambda}$-open set respectively.

\section{Proposition 3.2:}

1) [3]Every closed (resp.open) set is $\lambda$-closed (resp. $\lambda$-open) set.

2) Every $\lambda$-closed (resp. $\lambda$-open) set is $\Lambda^{\lambda}$-closed (resp. $\Lambda^{\lambda}$-open) set.

Definition 3.3:A function $f:(\mathrm{X}, \tau) \rightarrow(\mathrm{Y}, \sigma)$ is called :

1) $\Lambda$-g-continuous if $f^{-1}(\mathrm{~V})$ is $\Lambda$-g-open in $(\mathrm{X}, \tau)$, for every open set $\mathrm{V}$ in $(\mathrm{Y}, \sigma)$.

2) $\Lambda^{\lambda}$-continuous if $f^{-1}(\mathrm{~V})$ is $\Lambda^{\lambda}$-open in $(\mathrm{X}, \tau)$, for every open set $\mathrm{V}$ in $(\mathrm{Y}, \sigma)$.

3) $\quad \Lambda^{\lambda}-\lambda$-continuous if $f^{-1}(\mathrm{~V})$ is $\Lambda^{\lambda}$-open in $(\mathrm{X}, \tau)$, for every $\lambda$-open set $\mathrm{V}$ in $(\mathrm{Y}, \sigma)$.

4) $\Lambda^{\lambda}$-irresolute if $f^{-1}(\mathrm{~V})$ is $\Lambda^{\lambda}$-open in $(\mathrm{X}, \tau)$, for every $\Lambda^{\lambda}$-open set $\mathrm{V}$ in $(\mathrm{Y}, \sigma)$.

5) $\Lambda^{\lambda *}$-open if $f(V)$ is $\Lambda^{\lambda}$-open in $(Y, \sigma)$, for every $\Lambda^{\lambda}$-open set $V$ in $(X, \tau)$.

Definition 3.4: Let $(X, \tau)$ be a space and $A \subset X$. A Point $x \in X$ is called

$\Lambda^{\lambda}$-cluster point of $A$ if for every $\Lambda^{\lambda}$-open set $U$ of $X$ containing $x, A \cap U \neq \phi$. The set of all $\Lambda^{\lambda}$-cluster points is called the $\Lambda^{\lambda}$ - closure of $A$ and is denoted by $C l^{\Lambda^{\lambda}}(A)$. 
Theorem 3.5: If $f:(\mathrm{X}, \tau) \rightarrow(\mathrm{Y}, \sigma)$ is $\Lambda^{\lambda}$ - irresolute then the map $f$ is

$\Lambda^{\lambda}$ - continuous.

Proof: Let $f$ be $\Lambda^{\lambda}$ - irresolute Let $V$ be an open set in $(Y, \sigma)$. By Proposition 3.2, $V$ is $\Lambda^{\lambda}$-open in $(Y, \sigma)$. Since $f$ is $\Lambda^{\lambda}$ - irresolute, $f^{-1}(V)$ is $\Lambda^{\lambda}$-open in $(X, \tau)$. Hence $f$ is $\Lambda^{\lambda}$ - continuous.

Proposition 3.6: Let $A$ and $B$ be a subset of a topological space $(X, \tau)$. The following properties hold:

(1) $A \subset C l^{\Lambda^{\lambda}}(A) \subset C l^{\lambda}(A)$

(2) $C l^{\Lambda^{\lambda}}(\mathrm{A})=\cap\left\{\mathrm{F} \in \Lambda^{\lambda} \mathrm{C}(\mathrm{X}, \tau) / \mathrm{A} \subset \mathrm{F}\right\}$

(3) If $\mathrm{A} \subset \mathrm{B}$ then $\mathrm{Cl} l^{\Lambda^{\lambda}}(\mathrm{A}) \subset C l^{\Lambda^{\lambda}}(\mathrm{B})$

(4) $A$ is $\Lambda^{\lambda}$ - closed if and only if $A=C l^{\Lambda^{\lambda}}(A)$.

(5) $C l^{\Lambda^{\lambda}}(\mathrm{A})$ is $\Lambda^{\lambda}$-closed.

\section{Proof:}

(1) Let $x \notin C l^{\Lambda^{\lambda}}(A)$. Then $x$ is not a $\Lambda^{\lambda}$-cluster point of $\mathrm{A}$. So there exists a $\Lambda^{\lambda}$-open set $\mathrm{U}$ containing $x$ such that $A \cap U=\phi$ and hence $x \notin A$.

Then $C l^{\Lambda^{\lambda}}(A) \subset C l^{\lambda}(A)$ follows from Proposition 3.2.

(2) Suppose $x \in \cap\left\{F / A \subset F\right.$ and $F$ is $\Lambda^{\lambda}-$ closed $\}$. Let $U$ be a $\Lambda^{\lambda}$-open set containing $x$ such that $A \cap U=\phi$.Andso $A \subset X-U$. But $\mathrm{X}-U$ is $\Lambda^{\lambda}$-closed and hence $C l^{\Lambda^{\lambda}}(A) \subset X-U$. Since $x \notin X-U$, we obtain $x \notin C l^{\Lambda^{\lambda}}(A)$

which is contrary to the hypothesis. Hence $C l^{\Lambda^{\lambda}}(A) \supset \cap\left\{F / A \subset F\right.$ and $F$ is $\Lambda^{\lambda}-$ closed $\}$.

Suppose that $x \in C l^{\Lambda^{\lambda}}(A)$, i.e., that every $\Lambda^{\lambda}$-open set of $\mathrm{X}$ containing $\mathrm{x}$ meets A. If $x \notin \cap\left\{F / A \subset F\right.$ and $F$ is $\Lambda^{\lambda}-$ closed $\}$, then there exists a $\Lambda^{\lambda}$-closed set

F of $\mathrm{X}$ such that $A \subset F$ and $x \notin F$. Therefore $x \in X-F \in \Lambda^{\lambda} O(X, \tau)$. Hence $\mathrm{X}-\mathrm{F}$ is a $\Lambda^{\lambda}$-open set of $X$ containing $x$, but $(X-F) \cap A=\phi$. But this is a contradiction. Hence $C l^{\Lambda^{\lambda}}(A) \subset \cap\left\{F / A \subset F\right.$ and $F$ is $\Lambda^{\lambda}-$ closed $\}$. Thus, 
$C l^{\Lambda^{\lambda}}(A)=\cap\left\{F / A \subset F\right.$ and $F$ is $\Lambda^{\lambda}-$ closed $\}$.

(3) Let $x \notin C l^{\Lambda^{\lambda}}(B)$. Then there exists a $\Lambda^{\lambda}$-open set $\vee$ containing $x$ such that $B \cap V=\phi$. Since $A \subset B, A \cap V=\phi$ and hence $\mathrm{x}$ is not a $\Lambda^{\lambda}$-cluster point of A.

Therefore $x \notin C l^{\Lambda^{\lambda}}(A)$.

(4) Let $\mathrm{A}$ is $\Lambda^{\lambda}$ closed. Let $x \notin A$ Then $\mathrm{x}$ belongs to the $\Lambda^{\lambda}$-open $\mathrm{X}-\mathrm{A}$. Then a $\Lambda^{\lambda}$-open set X-A containing $x$ and $A \cap(X-A)=\phi$. Hence $x \notin C l^{\Lambda^{\lambda}}(A)$. By

(1),we get $A=C l^{\Lambda^{\lambda}}(A)$. Conversely, Suppose $A=C l^{\Lambda^{2}}(A)$. By (2) $A=\cap\left\{F \in \Lambda^{\lambda} C(X, \tau) / A \subset F\right\}$. Hence $A$ is $\Lambda^{\lambda}$-closed .

(5) By (1) and (3), we have $C l^{\Lambda^{\lambda}}(A) \subset C l^{\Lambda^{\lambda}}\left(C l^{\Lambda^{\lambda}}(A)\right)$. Let $x \in C l^{\Lambda^{\lambda}}\left(C l^{\Lambda^{\lambda}}(A)\right)$. Hence $\mathrm{x}$ is a $\Lambda^{\lambda}$-cluster point of $C l^{\Lambda^{\lambda}}(A)$. That implies for every $\Lambda^{\lambda}$-open set $U$ containing $x, C l^{\Lambda^{\lambda}}(A) \cap U \neq \phi$. Let $p \in C l^{\Lambda^{\lambda}}(A) \cap U$. Then for every $\Lambda^{\lambda}$-open set $G$ containing $p, A \cap G \neq \phi$, since $p \in C l^{\Lambda^{\lambda}}(A)$. Since $U$ is $\Lambda^{\lambda}$-open and $x, p \in U, A \cap U \neq \phi$. Hence $x \in C l^{\Lambda^{\lambda}}(A)$. Hence $C l^{\Lambda^{\lambda}}(A)=C l^{\Lambda^{\lambda}}\left(C l^{\Lambda^{\lambda}}(A)\right)$.By $(4), C l^{\Lambda^{\lambda}}(A)$ is $\Lambda^{\lambda}$-closed.

Definition 3.7:A subset $\mathrm{A}$ of a topological space $(X, \tau)$ is said to be $\lambda$-locally closed if $A=S \cap P$, where $\mathrm{S}$ is $\lambda$-open in $\mathrm{X}$ and $\mathrm{P}$ is $\lambda$-closed in $\mathrm{X}$.

Lemma 3.8: Let $\mathrm{A}$ be $\Lambda^{\lambda}$-closed subset of a topological space $(X, \tau)$. Then we have,

1) $A=T \cap C l^{\lambda}(A)$, where $\mathrm{T}$ is a $\Lambda_{*}^{\lambda}$-set.

2) $A=A^{\Lambda_{*}^{\lambda}} \cap C l^{\lambda}(A)$

Lemma 3.9: A subset $A \subseteq(X, \tau)$ is $\Lambda-g-$ closed iff $C l^{\lambda}(A) \subseteq A^{\Lambda_{*}^{\lambda}}$.

Proposition3.10: For a subset A of a topological space the following conditions are equivalent.

(1) $A$ is $\lambda$ - closed.

(2) $\mathrm{A}$ is $\Lambda$-g-closed and $\lambda$-locally closed

(3) $\mathrm{A}$ is $\Lambda$-g-closed and $\Lambda^{\lambda}$ - closed.

Proof: $(1) \Rightarrow(2)$ Every $\lambda$-closed set is both $\Lambda$-g-closed and $\lambda$-locally closed.

$(2) \Rightarrow(3)$ This is obvious from the fact that every $\lambda$-locally closed is a $\Lambda^{\lambda}$-closed. 
(3) $\Rightarrow$ (1) $\mathrm{A}$ is $\Lambda$-g-closed, so by Lemma $3.9, C l^{\lambda}(A) \subseteq A^{\Lambda_{*}^{\lambda}}$. A is $\Lambda^{\lambda}$-closed, so by Lemma 3.8 , $A=A^{\Lambda_{*}^{\lambda}} \cap C l^{\lambda}(A)$. Hence $A=C l^{\lambda}(A)$, i.e., $\mathrm{A}$ is $\lambda$-closed.

\section{4. $\Lambda^{\wedge}$-closed Maps}

In this section, we introduce $\Lambda_{*}^{\lambda}$ - closed maps. $\Lambda_{*}^{\lambda}$ - open maps, $\Lambda^{\lambda}$-closed maps, $\Lambda^{\lambda}$-open maps, $\Lambda^{\lambda *}$-closed maps and $\Lambda^{\lambda *}$-open maps.

\section{Definition 4.1:}

A map $f:(\mathrm{X}, \tau) \rightarrow(\mathrm{Y}, \sigma)$ is said to be a $\Lambda_{*}^{\lambda}$-closed map if the image of every closed set in $(\mathrm{X}, \tau)$ is $V_{*}^{\lambda}$-set in $(\mathrm{Y}, \sigma)$.

\section{Example 4.2:}

(a) Let $X=Y=\{a, b, c, d\}, \tau=\{\phi, X,\{a, b\},\{a, b, c\}\}$ and $\sigma=\{\phi, Y,\{a\},\{a, b\},\{a, c, d\}\}$. Define a map $f:(\mathrm{X}, \tau) \rightarrow(Y, \sigma)$ by $f(\mathrm{a})=\mathrm{c}, f(\mathrm{~b})=\mathrm{d}, f(\mathrm{c})=\mathrm{b}$ and $f(\mathrm{~d})=\mathrm{a}$.

Then $f$ is a $\Lambda_{*}^{\lambda}$-closed.

(b) Let $X=Y=\{a, b, c, d\}, \tau=\{\phi, X,\{a\},\{b\},\{a, b\}\}$ and $\sigma=\{\phi, Y,\{d\},\{b, c, d\}\}$. Let $f:(X, \tau) \rightarrow(Y, \sigma)$ be the identity map $f(\{c, d\})=\{c, d\}$ is not a $V_{*}^{\lambda}$-set. Hence $f$ is not a $\Lambda_{*}^{\lambda}$-closed map.

Definition 4.3: A map $f:(\mathrm{X}, \tau) \rightarrow(Y, \sigma)$ is said to be $\Lambda^{\lambda}$-closed if the image of every closed set in $(\mathrm{X}, \tau)$ is $\Lambda^{\lambda}$-closed in $(Y, \sigma)$.

\section{Example 4.4:}

(a) Let $X=Y=\{a, b, c, d, e\}, \tau=\{\phi, X,\{a\},\{d, e\},\{a, d, e\},\{b, c, d, e\}\}$ and $\sigma=\{\phi, Y,\{b, c\},\{b, c, d\},\{a, b, d, e\}\}$. Define a map $f:(X, \tau) \rightarrow(Y, \sigma)$ by $f(a)=\mathrm{d}, f(\mathrm{~b})=\mathrm{e}, f(\mathrm{c})=\mathrm{a}, f(\mathrm{~d})=\mathrm{c}$ and $f(\mathrm{e})=\mathrm{b}$. Then $f$ is a $\Lambda^{\lambda}$-closed map.

(b) Let $X=Y=\{a, b, c, d\}, \tau=\{\phi, X,\{a, b\},\{a, b, c\}\}$ and $\sigma=\{\phi, Y,\{a\},\{b, c\},\{a, b, c\},\{b, c, d\}\}$. Let $f:(X, \tau) \rightarrow(Y, \sigma)$ be an identity map. $f(\{c, d\})=\{c, d\}$ is not a $\Lambda^{\lambda}$-closed set. Hence $f$ is not a $\Lambda^{\lambda}$ - closed map.

Definition 4.5: A map $f:(\mathrm{X}, \tau) \rightarrow(Y, \sigma)$ is said to be $\Lambda$-g-closed if the image of every closed set in $(\mathrm{X}, \tau)$ is $\Lambda$-g-closed in $(Y, \sigma)$. 


\section{Example 4.6:}

(i) Let $X=Y=\{a, b, c, d\}, \tau=\{\phi, X,\{d\},\{a, d\},\{c, d\},\{a, c, d\}\}$ and $\sigma=\{\phi, Y,\{a, b\},\{c, d\}\}$. Define an identity map $f: X \rightarrow Y$ is $\Lambda$-g-closed.

(ii) Let $X=Y=\{a, b, c, d\}, \tau=\{\phi, X,\{a\},\{a, c\},\{a, c, d\}\}$ and $\sigma=\{\phi, Y,\{d\},\{c, d\},\{a, c, d\}\}$. Define a map $f:(X, \tau) \rightarrow(Y, \sigma)$ by $f(a)=d$, $f$ b) $=$ b, $f(c)=a, f(d)=c$. But $\{b, c\}$ is not a $\Lambda$-g-closed. Hence $f$ is not $\Lambda$-g-closed.

\section{Definition 4.7:}

A map $f:(\mathrm{X}, \tau) \rightarrow(Y, \sigma)$ is said to be $\Lambda^{\lambda}-\lambda$-closed if the image of every $\lambda$-closed set in $(\mathrm{X}, \tau)$ is $\Lambda^{\lambda}$-closed in $(Y, \sigma)$.

\section{Example 4.8:}

The function $f$ which is defined in example $4.2(a)$ is $\Lambda^{\lambda}-\lambda$-closed.

\section{Proposition 4.9:}

Every $\Lambda^{\lambda}-\lambda$-closed map is a $\Lambda^{\lambda}$-closed map.

\section{Proof:}

Let $f:(\mathrm{X}, \tau) \rightarrow(Y, \sigma)$ be a $\Lambda^{\lambda}-\lambda$-closed map. Let $\mathrm{B}$ be a closed set in $(\mathrm{X}, \tau)$ and hence $\mathrm{B}$ is a $\lambda-$ closed set in $(\mathrm{X}, \tau)$. By assumption $f(\mathrm{~B})$ is $\Lambda^{\lambda}$-closed in $(Y, \sigma)$. Hence $f$ is a $\Lambda^{\lambda}$-closed map.

\section{Theorem 4.10:}

Let $f:(\mathrm{X}, \tau) \rightarrow(Y, \sigma)$ and $\mathrm{g}:(\mathrm{Y}, \sigma) \rightarrow(\mathrm{Z}, \eta)$ be two mappings such that their composition gof: $(X, \tau) \rightarrow(Z, \eta)$ be a $\Lambda^{\lambda}$-closed mapping. Then the following statements are true if

1) $f$ is continuous and surjective then $\mathrm{g}$ is $\Lambda^{\lambda}$-closed.

2) $\mathrm{g}$ is $\Lambda^{\lambda}$-irresolute and injective then $f$ is $\Lambda^{\lambda}$-closed.

3) $f$ is g-continuous, surjective and $(X, \tau)$ is a $T_{1 / 2}$ - space then $\mathrm{g}$ is $\Lambda^{\lambda}$-closed

\section{Proof:}

1) Let $\mathrm{A}$ be a closed set in $(Y, \sigma)$. Since $f$ is continuous, $f^{-1}(\mathrm{~A})$ is closed in $(X, \tau)$ and since gof is $\Lambda^{\lambda}$-closed, (gof) $\left(f^{-1}(\mathrm{~A})\right)$ is $\Lambda^{\lambda}$-closed in $(Z, \eta)$. i.e., $\mathrm{g}(\mathrm{A})$ is $\Lambda^{\lambda}$-closed in $(Z, \eta)$. 
2) Let $B$ be a closed set of $(X, \tau)$. Since $g$ of is $\Lambda^{\lambda}$-closed, (gof) (B) is $\Lambda^{\lambda}$-closed in $(Z, \eta)$. Since $g$ is $\Lambda^{\lambda}$-irresolute $\mathrm{g}^{-1}((g \circ f)(B))$ is $\Lambda^{\lambda}$-closed in $(Y, \sigma)$.i.e., $f(B)$ is $\Lambda^{\lambda}$-closed in $(Y, \sigma)$, Since $g$ is injective. Thus $f$ is a $\Lambda^{\lambda}$-closed map.

3) Let $\mathrm{A}$ be a closed set of $(\mathrm{Y}, \sigma)$. Since $f$ is g-continuous, $f^{-1}(\mathrm{~A})$ is $\mathrm{g}$ - closed in $(X, \tau)$. Since $(X, \tau)$ is a $T_{1 / 2}$-space, $f^{-1}(\mathrm{~A})$ is closed in $(X, \tau)$ and so as in (i), $\mathrm{g}$ is a $\Lambda^{\lambda}$-closed map.

Theorem 4.11:Let $f:(X, \tau) \rightarrow(\mathrm{Y}, \sigma)$ and $\mathrm{g}:(\mathrm{Y}, \sigma) \rightarrow(\mathrm{Z}, \eta)$ be two mappings such that their composition gof: $(X, \tau) \rightarrow(Z, \eta)$ be a $\Lambda^{\lambda}-\lambda$-closed mapping. Then the following is true if

1) $f$ is $\lambda$-irresolute and surjective then $\mathrm{g}$ is $\Lambda^{\lambda}-\lambda$-closed.

2) $\mathrm{g}$ is $\Lambda^{\lambda}$-irresolute and injective then $f$ is $\Lambda^{\lambda}-\lambda$-closed.

\section{Proof:}

1) Let $\mathrm{A}$ be a $\lambda$-closed set of $(\mathrm{Y}, \sigma)$. Since $f$ is $\lambda$ - irresolute, $f^{-1}(\mathrm{~A})$ is $\lambda$-closed in $(X, \tau)$ and since gof is $\Lambda^{\lambda}-\lambda$-closed, (gof) $\left(f^{-1}(\mathrm{~A})\right)$ is $\Lambda^{\lambda}$-closed in $(Z, \eta)$.

i.e., $g(A)$ is $\Lambda^{\lambda}$-closed in $(Z, \eta)$. Hence $g$ is $\Lambda^{\lambda}-\lambda$-closed.

2)Let $\mathrm{B}$ be a $\lambda$-closed set of $(X, \tau)$. Since gof is $\Lambda^{\lambda}-\lambda$-closed, (gof) (B) is $\Lambda^{\lambda}$ - closed in $(\mathrm{Z}, \eta)$. Since $\mathrm{g}$ is $\Lambda^{\lambda}$-irresolute $\mathrm{g}^{-1}((\mathrm{gof})(\mathrm{B}))$ is $\Lambda^{\lambda}$-closed in $(\mathrm{Y}, \sigma)$.i.e., $f(\mathrm{~B})$ is

$\Lambda^{\lambda}$-closed in $(Y, \sigma)$, since $g$ is injective. Thus $f$ is $\Lambda^{\lambda}-\lambda$-closed map.

\section{Definition 4.12:}

A map $f:(X, \tau) \rightarrow(Y, \sigma)$ is said to be a $\Lambda^{\lambda}$-open map if the image $f(\mathrm{~A})$ is $\Lambda^{\lambda}$-open in $(\mathrm{Y}, \sigma)$ for each open set $\mathrm{A}$ in $(X, \tau)$.

\section{Proposition: 4.13:}

For any bijection $f:(X, \tau) \rightarrow(Y, \sigma)$, the following statements are equivalent.

1) $f^{-1}:(\mathrm{Y}, \sigma) \rightarrow(X, \tau)$ is $\Lambda^{\lambda}$-continuous.

2) $f$ is a $\Lambda^{\lambda}$-open map and

3) $f$ is a $\Lambda^{\lambda}$-closed map.

\section{Proof:}

$(1) \Rightarrow(2)$ : Let $U$ be an open set of $(X, \tau)$. By assumption $\left(f^{-1}\right)^{-1}(U)=f(U)$

is $\Lambda^{\lambda}$-open in $(\mathrm{Y}, \sigma)$ and so $f$ is $\Lambda^{\lambda}$-open. 
$(2) \Rightarrow(3)$ : Let $F$ be a closed set of $(X, \tau)$. Then $X-F$ is open in $(X, \tau)$. By

assumption, $f(\mathrm{X}-\mathrm{F})$ is $\Lambda^{\lambda}$-open in $(\mathrm{Y}, \sigma)$ and therefore

$f(\mathrm{X}-\mathrm{F})=(\mathrm{Y}-f(\mathrm{~F}))$ is $\Lambda^{\lambda}$-open in $(\mathrm{Y}, \sigma)$ and therefore $f(\mathrm{~F})$ is

$\Lambda^{\lambda}$-closed in $(Y, \sigma)$. Hence $f$ is $\Lambda^{\lambda}$-closed.

$(3) \Rightarrow(1)$ :Let $\mathrm{F}$ be a closed set of $(X, \tau)$. By assumption, $f(\mathrm{~F})$ is $\Lambda^{\lambda}$-closed in $(\mathrm{Y}, \sigma)$. But $f(\mathrm{~F})=\left(f^{-1}\right)^{-1}(\mathrm{~F})$ and therefore $f^{-1}$ is $\Lambda^{\lambda}$-continuous on $\mathrm{Y}$.

Definition 4.14: A map $f:(X, \tau) \rightarrow(Y, \sigma)$ is said to be a $\Lambda^{\lambda}-\lambda$-open map if the image $f$ $(\mathrm{A})$ is $\Lambda^{\lambda}$-open in $(\mathrm{Y}, \sigma)$ for each $\lambda$-open set $\mathrm{A}$ in $(X, \tau)$.

\section{Proposition: 4.15:}

For any bijection $f:(X, \tau) \rightarrow(Y, \sigma)$, the following statements are equivalent.

1) $f^{-1}:(\mathrm{Y}, \sigma) \rightarrow(X, \tau)$ is $\Lambda^{\lambda}-\lambda$-continuous.

2) $f$ is a $\Lambda^{\lambda}-\lambda$-open map and

3) $f$ is a $\Lambda^{\lambda}-\lambda$-closed map

\section{Proof:}

$(1) \Rightarrow(2)$ Let $U$ be an $\lambda$-open set of $(X, \tau)$. By assumption $\left(f^{-1}\right)^{-1}(U)=f(U)$

is $\Lambda^{\lambda}$-open in $(\mathrm{Y}, \sigma)$ and so $f$ is $\Lambda^{\lambda}-\lambda$-open.

$(2) \Rightarrow(3)$ Let $F$ be a $\lambda$ - closed set of $(X, \tau)$. Then $X-F$ is $\lambda$ - open in $(X, \tau)$. By assumption, $f(\mathrm{X}-\mathrm{F})$ is $\Lambda^{\lambda}$-open in $(\mathrm{Y}, \sigma)$ i.e., $f(\mathrm{X}-\mathrm{F})=\mathrm{Y}-f(\mathrm{~F})$ is $\Lambda^{\lambda}$-open in $(\mathrm{Y}, \sigma)$ and there $f(\mathrm{~F})$ is $\Lambda^{\lambda}$-closed in $(\mathrm{Y}, \sigma)$. Hence $f$ is $\Lambda^{\lambda}$-closed.

(3) $\Rightarrow(1)$ Let $\mathrm{F}$ be a $\lambda$-closed set in $(X, \tau)$. By assumption. $f(\mathrm{~F})$ is

$\Lambda^{\lambda}-\lambda$ - closed in $(\mathrm{Y}, \sigma)$. But $\mathrm{f}(\mathrm{F})=\left(f^{-1}\right)^{-1}(\mathrm{~F})$ and therefore $f^{-1}$ is

$\Lambda^{\lambda}-\lambda$ - continuous on $Y$.

Definition 4.16: (i) A map $f:(X, \tau) \rightarrow(Y, \sigma)$ is said to be a $\lambda^{*}$-closed map if the image of $f(\mathrm{~A})$ is $\lambda$-closed in $(\mathrm{Y}, \sigma)$ for every $\lambda$-closed set $\mathrm{A}$ in $(X, \tau)$.

(ii) A map $f:(X, \tau) \rightarrow(Y, \sigma)$ is said to be a $\Lambda^{\lambda *}$-closed map if the image $f(\mathrm{~A})$ is $\Lambda^{\lambda}$-closed in $(\mathrm{Y}, \sigma)$ for every $\Lambda^{\lambda}$-closed set $\mathrm{A}$ in $(X, \tau)$. 
(iii) A map $f:(X, \tau) \rightarrow(Y, \sigma)$ is said to be a $\Lambda$ - gc-closed if the image $f$ (A)

is $\Lambda-g$-closed in $(\mathrm{Y}, \sigma)$ for every $\Lambda-g$-closed set $\mathrm{A}$ in $(X, \tau)$.

\section{Example: 4.17}

(i)

$$
\text { Let } X=Y=\{a, b, c, d, e\}, \tau=\{\phi, X,\{a\},\{a, b, c\}\} \text { and } \sigma=\{\phi, Y,\{a\},\{b\},\{a, b\}\} \text {. Define }
$$

a $\operatorname{map} f:(X, \tau) \rightarrow(Y, \sigma)$ by $f(a)=a, f(b)=c, f(c)=d, f(d)=b$ and $f(e)=e$. Then $f$ is $\lambda^{*}$-closed as well as $\Lambda^{\lambda *}$-closed.

\section{Remark 4.18:}

Since every closed set is a $\Lambda^{\lambda}$-closed set we have every $\Lambda^{\lambda *}$ - closed map is a $\Lambda^{\lambda}$-closed map.

The converse is not true in general as seen from the following example.

\section{Example 4.19:}

(i)

$$
\text { Let } X=Y=\{a, b, c, d\}, \tau=\{\phi, X,\{a\},\{a, b, c\}\} \text { and }
$$

$\sigma=\{\phi, Y,\{a\},\{b\},\{a, b\}\}$. Defineamap $f:(X, \tau) \rightarrow(Y, \sigma)$ by

$f(a)=a, f(b)=b, f(c)=d, f(d)=c$. Then $f$ is $\Lambda^{\lambda}$-closed but not $\Lambda^{\lambda^{*}}$-closed, because for the $\Lambda^{\lambda}$-closed set $\{\mathrm{d}\}$ in $(X, \tau) f(\{d\})=c$ which is not a $\Lambda^{\lambda}$-closed set in $(Y, \sigma)$.

Proposition 4.20: For any bijection $f:(X, \tau) \rightarrow(Y, \sigma)$ the following are equivalent:

$$
f^{-1}:(Y, \sigma) \rightarrow(X, \tau) \text { is } \Lambda^{\lambda} \text {-irresolute }
$$

(ii) $\quad f$ is a $\Lambda^{\lambda}$ c-open map and

(iii) $\quad f$ is a $\Lambda^{\lambda}$ c-closed map.

Proof: Similar to proposition 4.13.

\section{Definition 4.21:}

Let $\mathrm{A}$ be a subset of $\mathrm{X}$. A mapping $r: X \rightarrow A$ is called a $\Lambda^{\lambda}-$ continuous retraction if $r$ is a $\Lambda^{\lambda}$ continuous and the restriction if $r$ is a $\Lambda^{\lambda}$ - continuous and the restriction $r_{A}$ is the identity mapping on $A$.

Definition 4.22: A topological space $(X, \tau)$ is called a $\Lambda^{\lambda}$ - Hausdorff if for each pair $x, y$ of distinct points of $\mathrm{X}$ there exists $\Lambda^{\lambda}$-neighborhoods $U_{1}$ and $U_{2}$ of $x$ and $y$, respectively, that are disjoint. 


\section{Theorem 4.24:}

Let $\mathrm{A}$ be a subset of $\mathrm{X}$ and $r: X \rightarrow A$ be a $\Lambda^{\lambda}$-continuous retraction. If $\mathrm{X}$ is $\Lambda^{\lambda}$-Hausdorff, then $A$ is a $\Lambda^{\lambda}$-closed set of $X$.

\section{Proof:}

Suppose that $\mathrm{A}$ is not $\Lambda^{\lambda}$-closed. Then there exists a point $x$ in $\mathrm{X}$ such that $x \in c \ell^{\Lambda^{\lambda}}(A)$ but $x \notin A$. It follows that $r(x) \neq x$ because $r$ is $\Lambda^{\lambda}$-continuous retraction. Since $\mathrm{X}$ is $\Lambda^{\lambda}$-Hausdroff, there exists disjoint $\Lambda^{\lambda}$-open sets $U$ and $\mathrm{V}$ in $\mathrm{X}$ such that $x \in U$ and $r(x) \in V$. Now let $W$ be an arbitrary $\Lambda^{\lambda}$-neighborhood of $x$. Then $W \cap U$ is a $\Lambda^{\lambda}$-neighborhood of $x$. Since $x \in C \ell^{\Lambda^{\lambda}}(A)$, we have $(W \cap U) \cap A \neq \phi$. Therefore there exists a point $y$ in $W \cap U \cap A$. Since $y \in A$, We have $r(y)=y \in U$ and hence $r(y) \notin V$. This implies that $r(W) \not \subset V$ because $y \in W$. This is contrary to the $\Lambda^{\lambda}$-continuity of $r$. Consequently, $\mathrm{A}$ is a $\Lambda^{\lambda}$-closed set of $\mathrm{X}$.

\section{Theorem 4.25}

Let $\{X i: i \in I\}$ be any family of topological space. If $f: X \rightarrow \pi x i$ is a $\Lambda^{\lambda}$-continuous mapping, then $P_{r_{i}}$ of : $X \rightarrow X i$ is $\Lambda^{\lambda}$-continuous for each $i \in I$, where $P_{r_{i}}$ is the projection of $\pi x_{j}$ on to $X_{i}$.

Proof: We shall consider a fixed $i \in I$. Suppose $\mathrm{U}_{\mathrm{i}}$ is an arbitrary open set in X. Then $P_{r i}^{-1}\left(U_{i}\right)$ is open in $\pi X i$. Since $f$ is $\Lambda^{\lambda}$-continuous, we have $f^{-1}\left(p_{r_{i}}{ }^{-1}\left(U_{i}\right)\right)=\left(p_{r_{i}} \circ f\right)^{-1}\left(U_{i}\right) \quad \Lambda^{\lambda}$-open in X. Therefore $P_{r_{i}} \circ f$ is $\Lambda^{\lambda}$-continuous.

\section{Proposition 4.26:}

A mapping $f:(X, \tau) \rightarrow(Y, \sigma)$ is $\Lambda^{\lambda}$-closed if and only if $C l^{\Lambda^{\lambda}}(f(A)) \subset f(C l(A))$ for every subset $\mathrm{A}$ of $(X, \tau)$.

Proof: Suppose that $f$ is $\Lambda^{\lambda}$-closed and $A \subset X$. Then $f(\mathrm{Cl}(\mathrm{A}))$ is $\Lambda^{\lambda}$-closed in $(Y, \sigma)$. We have $f(A) \subset f(C l(A))$ and by 3.6, $C l^{\Lambda^{\lambda}}(f(A)) \subset C l^{\Lambda^{\lambda}}(f(C l(A)))=f(C l(A))$.Conversely, let $\mathrm{A}$ be any closed set in $(X, \tau)$. By hypothesis and proposition 3.6, we have $\mathrm{A}=\mathrm{Cl}(\mathrm{A})$ and so $f(A)=f(C l(A)) \supset C l^{\Lambda^{\lambda}}(f(A))$. i.e., $f(A)$ is $\Lambda^{\lambda}$-closed and hence $f$ is $\Lambda^{\lambda}$-closed. 


\section{Theorem 4.27:}

A mapping $f:(X, \tau) \rightarrow(Y, \sigma)$ is $\lambda$-closed if and only if $f$ is both $\Lambda$-g-closed and $\Lambda^{\lambda}$-closed.

Proof: Let $V$ be a closed set in $(X, \tau)$. As $f$ is $\lambda$-closed, $f(V)$ is a $\lambda$-closed in $(Y, \sigma)$. By Proposition 3.10, $f(V)$ is a $\Lambda$-g-closed and $\Lambda^{\lambda}$-closed set. Hence $f$ is $\Lambda$-g-closed and $\Lambda^{\lambda}$ closed.

Conversely, let $\vee$ be closed in $(X, \tau)$. As $f$ is $\Lambda$-g-closed and $\Lambda^{\lambda}$-closed $f(V)$ is both $\Lambda$-g-closed and $\Lambda^{\lambda}$-closed set. Hence $f(V)$ is $\lambda$-closed by Proposition 3.10.

\section{5. $\Lambda^{\lambda}$-Homeomorphisms.}

In this section we introduce and study two new homeomorphisms namely $\Lambda^{\lambda}$-homeomorphism and $\Lambda^{\lambda^{*}}$ homeomorphism.

\section{Definition 5.1:}

A bijection $f:(X, \tau) \rightarrow(y, \sigma)$ is called $\lambda$-homeomorphism if $f$ is both

$$
\lambda \text {-continuous and } \lambda \text {-open. }
$$

Proposition 5.2: Every homeomorphism is a $\lambda$-homeomorphism.

Proof: Follows from definitions.

The converse of the Proposition 5.2 need not be true as see from the following example.

\section{Example 5.3:}

Let $X=Y=\{a, b, c, d\}, \tau=\{\phi, X,\{a\},\{a, b, c\}\}$ and $\sigma=\{\phi, Y,\{a\},\{b\},\{a, b\}\}$. Define a map $f:(X, \tau) \rightarrow(y, \sigma)$ by $f(a)=a, f(b)=c, f(c)=d$ and $f(d)=b$. Then $f$ is $\lambda$ - homeomorphism but not a homeomorphism, because it is not continuous.

Thus, the class of $\lambda$-homeomorphisms properly contains the class of homeomorphism.

Definition 5.4: A bijection $f:(X, \tau) \rightarrow(Y, \sigma)$ is called $\Lambda^{\lambda}$-homeomorphism if $f$ is both $\Lambda^{\lambda}$ continuous and $\Lambda^{\lambda}$-open.

Proposition 5.5: Every $\lambda$-homeomorphism is a $\Lambda^{\lambda}$-homeomorphism proof: Follows from definitions.

The converse of the Proposition 5.5 need not be true as seen from the following example.

\section{Example5.6:}

Let $X=Y=\{a, b, c, d\}, \tau=\{\phi, X,\{a, b\},\{a, b, c\}\}$ and $\sigma=\{\phi, Y,\{a\},\{a, b\},\{a, c, d\}\}$. Define a map $f:(X, \tau) \rightarrow(Y, \sigma)$ by $f(a)=c, f(b)=d, f(c)=a$ and $\quad f(d)=b . \quad$ Then $f$ is $\Lambda^{\lambda}-$ homeomorphism but not a homeomorphism. Because it is not a $\lambda$-continuous function. 
Thus, the class of $\Lambda^{\lambda}$-homeomorphisms properly contains the class of $\lambda$-homeomorphisms.

\section{Definition 5.7:}

A bijection $f:(X, \tau) \rightarrow(\mathrm{Y}, \sigma)$ is called $\Lambda^{\lambda}-\lambda$-homeomorphism if $f$ is both $\Lambda^{\lambda}-\lambda$-continuous and $\Lambda^{\lambda}-\lambda$-open.

\section{Proposition 5.8:}

Every $\Lambda^{\lambda}-\lambda$-homeomorphism is a $\Lambda^{\lambda}$-homeomorphism but not conversely.

Proof: Follows from definitions.

The converse of the Proposition 5.8 need not be true as seen from the following example.

\section{Example 5.9:}

The function $f$ in 5.3 is $\Lambda^{\lambda}$-homeomorphism but not $\Lambda^{\lambda}-\lambda$-homeomorphism. Because $f$ is not $\Lambda^{\lambda}-\lambda$-continuous.

Thus, the class of $\Lambda^{\lambda}$-homeomorphisms property contains the class of $\Lambda^{\lambda}-\lambda$-homeomorphisms.

Proposition 5.10: Let $f:(X, \tau) \rightarrow(\mathrm{Y}, \sigma)$ be a bijection $\Lambda^{\lambda}$-continuous map. Then the following statements are equivalent:

(i) $\quad f$ is a $\Lambda^{\lambda}$-open map

(ii) $f$ is a $\Lambda^{\lambda}$-homeomorphism

(iii) $\quad f$ is a $\Lambda^{\lambda}$-closed map.

Proof: (i) $\Leftrightarrow$ (ii) Follows from the definition.

(i) $\Leftrightarrow$ (iii) Follows from proposition 3.13 .

Proposition 5.11: Let $f:(X, \tau) \rightarrow(\mathrm{Y}, \sigma)$ be a bijection $\Lambda^{\lambda}-\lambda$-continuous map. Then the following statements are equivalent.

(i)

$$
f \text { is a } \Lambda^{\lambda}-\lambda \text {--open map }
$$

(ii) $f$ is a $\Lambda^{\lambda}-\lambda$--homeomorphism

(iii) $f$ is a $\Lambda^{\lambda}-\lambda$--closed map.

Proof: (i) $\Leftrightarrow$ (ii) Follows from the definition.

(i) $\Leftrightarrow$ (iii) Follows from proposition 3.15.

The composition of two $\Lambda^{\lambda}$-homeomorphism maps need not be a $\Lambda^{\lambda}$-homeomorphism as can be seen from the following example. 


\section{Example 5.12:}

Let

$$
\begin{aligned}
& X=Y=Z=\{a, b, c, d, e\}, \tau=\{\phi, X,\{b\},\{a, b\},\{b, c\},\{a, b, c\}\}, \\
& \sigma=\{\phi, Y,\{c, d\},\{b, c, d\},\{a, c, d\}\}
\end{aligned}
$$

and $\eta=\{\phi, Z,\{a\},\{b\},\{a, b\},\{b, c\},\{a, b, c\}\}$, Define a map $f:(X, \tau) \rightarrow(Y, \sigma)$ by

$$
f(a)=e, f(b)=b, f(c)=a, f(d)=c, f(e)=d \text { and } \quad g:(Y, \sigma) \rightarrow(Z, \eta) \quad \text { by }
$$

$g(a)=a, g(b)=b, g(c)=e, g(d)=d, g(e)=c . \quad$ Then $f$ and $g$ are $\Lambda^{\lambda}$-homeomorphisms but their composition gof $(X, \tau) \rightarrow(Z, \eta)$ is not a $\Lambda^{\lambda}$-homeomorphism, because $(g \circ f)^{-1}(\{a, b\})=\{b, c\}$ which is not a $\Lambda^{\lambda}$-open set in $(z, \eta)$. Therefore gof is not a $\Lambda^{\lambda}$ continuous map and so gof is not a $\Lambda^{\lambda}$-homeomorphism.

We next introduce a new class of maps called $\Lambda^{\lambda *}$-homeomorphisms which forms a sub class of $\Lambda^{\lambda}$-homeomorphisms. This class of maps is closed under composition of maps.

\section{Definition 5.13:}

A bijection $f:(X, \tau) \rightarrow(Y, \sigma)$ is said to be $\lambda^{*}$-homeomorphism if both

$f$ and $f^{-1}$ are $\lambda$-irresolute.

We denote the family of all $\lambda$-homeomorphisms (resp. $\lambda^{*}$-homeomorphism and homeomorphism) of a topological space $(X, \tau)$ on to itself by $\lambda-h(X, \tau)$. (resp. $\lambda^{*}-h(X, \tau)$ and $h(X, \tau)$.

\section{Proposition 5.14:}

Every $\lambda^{*}$-homeomorphism is a $\lambda$-homeomorphism but not conversely (i.e) for any space $(X, \tau)$, $\lambda^{*}-h(X, \tau) \subset \lambda-h(X, \tau)$.

\section{Proof:}

It follows from the fact that every $\lambda$-irresolute map is a $\lambda$-continuous map and the fact that $\lambda^{*}$-open map is $\lambda$-open map.

The function $f$ in example 5.3 is a $\lambda$-homeomorphism but not a $\lambda^{*}$-homeomorphism, since for the $\lambda$-closed set $\{a, b\}$ in $(Y, \sigma), f^{-1}(\{a, b\})=\{a, d\}$ which is not $\lambda$-closed in $(X, \tau)$. Therefore $f$ is not $\lambda$-irresolute and so $f$ is not a $\lambda^{*}$-homeomorphism.

\section{Definition 5.15:}

A bijection $f:(X, \tau) \rightarrow(Y, \sigma)$ is said to be $\Lambda^{\lambda *}$-homeomorphism if both $f$ and $f^{-1}$ are $\Lambda^{\lambda}$ irresolute. 
We denote the family of all $\Lambda^{\lambda}$-homeomorphisms (resp. $\Lambda^{\lambda *}$-homeomorphism) of a topological space $(X, \tau)$ on to itself by $\lambda^{*}-h(X, \tau)$. (resp. $\Lambda^{\lambda^{*}}-h(X, \tau)$.

\section{Proposition 5.16:}

Every $\Lambda^{\lambda *}$-homeomorphism is a $\Lambda^{\lambda}$-homeomorphism but not conversely (i.e) for any space $(X, \tau)$, $\Lambda^{\lambda^{*}}-h(X, \tau) \subset \Lambda^{\lambda}-h(X, \tau)$.

\section{Proof:}

Follows from theorem 3.5 and the fact that every $\Lambda^{\lambda *}$-open map is $\Lambda^{\lambda}$-open. The function $f$ in Example 5.4 is a $\Lambda^{\lambda}$-homeomorphism but not a $\Lambda^{\lambda *}$-homeomorphism, since for the $\Lambda^{\lambda}$-closed set $\{a, b . e\}$ in $(y, \sigma), f^{-1}(\{a, b, e\})=\{a, b, c\}$ which is not $\Lambda^{\lambda}$-closed set in $(X, \tau)$. Therefore $f$ is not $\Lambda^{\lambda}$-irresolute and so $f$ is not a $\Lambda^{\lambda *}$-homeomorphism.

\section{Theorem 5.17:}

If $f:(X, \tau) \rightarrow(Y, \sigma)$ and $g:(Y, \sigma) \rightarrow(Z, \eta)$ are $\Lambda^{\lambda *}$-homeomorphism, then their composition gof $:(X, \tau) \rightarrow(Z, \eta)$ is also $\Lambda^{\lambda *}$-homeomorphisms.

\section{Proof:}

Let $\mathrm{B}$ be a $\Lambda^{\lambda}$-open set in $(Z, \eta)$. Now, $(\text { gof })^{-1}(B)=f^{-1}\left(g^{-1}(B)=f^{-1}(C)\right.$, where $C=g^{-1}(B)$. By hypothesis, $\mathrm{C}$ is $\Lambda^{\lambda}$-open in $(Y, \sigma)$ and so again by hypothesis, $f^{-1}(C)$ is $\Lambda^{\lambda}$-open in $(X, \tau)$. Therefore gof is $\Lambda^{\lambda}$-irresolute. Also for a $\Lambda^{\lambda}$-open set $\mathrm{G}$ in $(X, \tau)$. We have $(g \circ f)(G)=g(f(G))=g(V)$, where $V=f(G)$. By hypothesis $f(G)$ is $\Lambda^{\lambda}$-open in $(Y, \sigma)$ and so again by hypothesis, $g(f(G))$ is $\Lambda^{\lambda}$-open in $(Z, \eta)$.i.e., $(g o f)(G)$ is $\Lambda^{\lambda}$-open in $(Z, \eta)$ and therefore $(\text { gof })^{-1}$ is $\Lambda^{\lambda}$-irresolute. Hence gof is a $\Lambda^{\lambda *}$-homeomorphism.

\section{Theorem 5.18:}

The set $\Lambda^{\lambda *}-h(X, \tau)$ is a group under the composition of maps.

\section{Proof:}

Define a binary operation *: $\Lambda^{\lambda *}-h(X, \tau) X \Lambda^{\lambda *}-h(X, \tau) \rightarrow \Lambda^{\lambda *}-h(X, \tau)$ by $f * g=g$ of for all $f, g \in \Lambda^{\lambda *}-h(X, \tau)$ and $\circ$ is the usual operation of composition of maps. Then by theorem 5.17, gof $\in \Lambda^{\lambda *}-h(X, \tau)$. We know that the composition of maps is associative and the identity map $I:(X, \tau) \rightarrow(X, \tau)$ belonging to $\Lambda^{2 *}-h(X, \tau)$ serves as the identity element. If 
$f \in \Lambda^{\lambda *}-h(X, \tau)$, then $f^{-1} \Lambda^{\lambda *}-h(X, \tau)$ such that $f o f^{-1}=f^{-1}$ of $=I$ and so inverse $\left(\Lambda^{\lambda *}-h(X, \tau), o\right)$ is a group under the operation of composition of maps.

\section{Theorem 5.19:}

Let $f:(X, \tau) \rightarrow(Y, \sigma)$ be a $\Lambda^{\lambda *}$ - homeomorphism. Then $f$ induces an isomorphism from the group $\Lambda^{\lambda *}-h(X, \tau)$ on to the group $\Lambda^{\lambda *}-h(Y, \sigma)$.

Proof:

Using the map $g$, we define a map $\wp_{g}: \Lambda^{\lambda *}-h(X, \tau) \rightarrow \Lambda^{\lambda *}-h(Y, \sigma)$ by $\wp_{g}(h)=$ gohog $^{-1}$ for every $h \in \Lambda^{\lambda *}-h(X, \tau)$. Then $\wp_{g}$ is a bijection. Further, for all $h_{1}, h_{2} \in \Lambda^{\lambda *}-h(X, \tau), \wp_{g}\left(h_{1} o h_{2}\right)=g o\left(h_{1} o h_{2}\right) o g^{-1}=\left(g o h_{1} o g^{-1}\right) o\left(g o h_{2} o g^{-1}\right)=\wp_{g}\left(h_{1}\right) o \wp_{g}\left(h_{2}\right)$.

Therefore, $\wp_{g}$ is a homeomorphism and so it is an isomorphism induced by $g$.

Theorem 5.20:

$\Lambda^{\lambda *}$-homeomorphisms is an equivalence relation in the collection of all topological spaces.

Proof: Reflexivity and symmetry are immediate and transitivity follows from Theorem 5.17.

\section{References}

[1] Francisco G.Arenas, J.Dontchev and M.Ganster (1997) On $\lambda$-sets and the dual of generalized continuity, $Q$ and $A$ in General topology 15: 3-13.

[2] M.Caldas, S.Jafari and T.Noiri (2008) On $\Lambda$-generalized closed sets in topological spaces, Acta Math.Hungar.118 (4): 337-343.

[3] M.Caldas, S.Jafari and G.Navalagi (2007) More on $\lambda$-closed set in toplological space, Revista Colombina de Mathematicas 41 (2): 355-369.

[4] H.Maki (1986) Generalized $\Lambda$-sets and the associated closure operator, in Speacial Issue in Commemoration of Prof.Kazusada Ikeda's Retirement pp.139-146.

[5] Maki H, P Sundaram and K Balachandran (1991) On generalized homeomorphisms in topological spaces, Bull. Fukuoka Univ. Ed. Part III 40: 13-21.

[6] Levine.N, Generalized closed sets in topology, Rend. Circ. Math. Palermo 19 (2): 89-96.

[7] Sundaram P (1991) Studies on generalizations of continuous maps in topological spaces, Ph.D. Thesis, Bharathiar University, Coimbatore. 